SOGANG-HEP-243/98

\title{
Entropy of $2+1$ dimensional de Sitter space in terms of brick wall method
}

\author{
Won Tae Kim* \\ Department of Physics and Basic Science Research Institute, \\ Sogang University, C.P.O. Box 1142, Seoul 100-611, Korea
}

(August 1998)

\begin{abstract}
We calculate the statistical entropy of a scalar field on the background of three-dimensional De Sitter space in terms of the brick wall method and finally derive the perimeter law of the entropy.
\end{abstract}

*Electronic address: wtkim@ccs.sogang.ac.kr 
Entropy of black holes has an universal area law [1] and the entropy of the well-known Schwarzschild black hole satisfies the area law by means of thermal radiation based on the quantum field theory [2]. On the other hand, 't Hooft has argued that when one calculates the black hole entropy, the modes of a quantum field in the vicinity of a black hole horizon should be cut off due to gravitational effects rather than infinitely piling up by suitably choosing a brick-wall cutoff just beyond the horizon [3]. Most of brick-wall calculations have been done for the asymptotically flat cases.

For $2+1$ dimensional anti-de Sitter space, Bañados, Teitelboim, and Zanelli (BTZ) have obtained a black hole solution which is asymptotically anti-de Sitter spacetime rather than asymptotically flat $\mathbb{4}$. The theormodynamic properties has been extensively studied in this black hole [5,6]. Recently the statistical entropy of the de Sitter(DS) space is studied in terms of Chern-Simons formulation [7]. The DS space has a cosmological horizon and asymptotically non-flat spacetime, furthermore the spacetime is bounded by the horizon as the two-dimensional cavity.

In this Brief Report, we shall calculate the entropy of a scalar field on the DS space background by using the brick wall method. As a result, the divergent entropy is obtained in the vicinity of horizon and by properly choosing the brick-wall cutoff we finally obtain the expected perimeter law.

Let us start with the following action

$$
I=\frac{1}{2 \pi} \int d^{3} x \sqrt{-g}\left[R-\frac{2}{l^{2}}\right]
$$

where $\Lambda=\frac{1}{l^{2}}$ is a cosmological constant. Then the classical equation of motion yields the DS metric as

$$
\begin{aligned}
& d s^{2}=-g(r) d t^{2}+\frac{1}{g(r)} d r^{2}+r^{2} d \theta^{2} \\
& g(r)=\left(1-\frac{r^{2}}{l^{2}}\right)
\end{aligned}
$$

The horizon is located at $r=l$ and our spacetime is defined within $0 \leq r \leq l$. The inverse 
of Hawking temperature is given by

$$
\beta_{H}=2 \pi l
$$

Let us now introduce a Klein-Gordon field equation on the DS background,

$$
\square \Phi=0,
$$

where we consider the massless case for simplicity. The Eq. (6) can be solved through the separation of variables and we can write the wave function as

$$
\Phi(r, \phi, t)=e^{-i E t} e^{i m \phi} R_{E m}(r)
$$

where $m$ is an azimuthal quantum number of the scalar field. Then the radial wave equation is written as

$$
\frac{1}{r g(r)} \partial_{r}\left[r g(r) \partial_{r} R_{E m}(r)\right]+k^{2}(r, m, E) R_{E m}(r)=0
$$

where the radial wave number is given by

$$
k^{2}(r, m, E)=\frac{1}{g^{2}(r)}\left[E^{2}-\frac{m^{2} g(r)}{r^{2}}\right]
$$

in the WKB approximation [3]. According to the semi-classical quantization rule, the radial wave number is quantized as

$$
\pi n_{r}(m, E)=\int_{L}^{r_{H}-\epsilon} d r k(r, m, E)
$$

under the brick wall boundary conditions: $\Phi=0$ at $r=L, r=r_{H}-\epsilon$. Note that $n_{r}$ is assumed to be a nonnegative integer, and $\epsilon$ and $L$ are ultraviolet and infrared regulators, respectively where $\epsilon>0$ and $0 \leq L \leq r_{H}-\epsilon$. In this range, the energy $E$ is always positive and the wave number $k$ is real.

The free energy at inverse temperature $\beta$ is given by

$$
e^{-\beta F}=\prod_{K}\left[1-e^{-\beta E_{K}}\right]^{-1}
$$


where $K$ represents the set of quantum numbers. By using Eq. (10), the free energy can be rewritten as

$$
\begin{aligned}
F & =\frac{1}{\beta} \sum_{K} \ln \left[1-e^{-\beta E_{K}}\right] \approx \frac{1}{\beta} \int d n_{r} \int d m \ln \left[1-e^{-\beta E}\right] \\
& =-\int d m \int d E \frac{n_{r}}{e^{\beta E}-1} \\
& =-\frac{1}{\pi} \int d m \int d E \frac{1}{e^{\beta E}-1} \int d r k(r, m, E),
\end{aligned}
$$

where we have taken the continuum limit in the first line and integrated by parts in the second line in Eq. (12). The explicit form of the free energy is given by

$$
\begin{aligned}
F & =-\frac{1}{\pi} \int_{0}^{\infty} d E \frac{1}{e^{\beta E}-1} \int_{L}^{r_{H}-\epsilon} d r \frac{1}{g(r)} \int d m \sqrt{E^{2}-\frac{m^{2} g(r)}{r^{2}}} \\
& =-\frac{1}{2} \int_{0}^{\infty} d E \frac{E^{2}}{e^{\beta E}-1} \int_{L}^{r_{H}-\epsilon} d r \frac{r}{g^{3 / 2}(r)} .
\end{aligned}
$$

Note that the integration with respect to angular variable $m$ is taken over values for which the square root is real. Performing the remaining integrations, the free energy is written by

$$
F=-\frac{\zeta(3) l^{3}}{2 \beta^{3}}\left(\frac{1}{\sqrt{l^{2}-\left(r_{H}-\epsilon\right)^{2}}}-\frac{1}{\sqrt{l^{2}-L^{2}}}\right) .
$$

Let us now evaluate the entropy for the massless field, which can be obtained from the free energy (14) at the Hawking temperature, then the entropy is

$$
\begin{aligned}
S & =\left.\beta^{2} \frac{\partial F}{\partial \beta}\right|_{\beta=\beta_{H}} \\
& =4 \pi a\left(\frac{l}{\sqrt{l^{2}-\left(r_{H}-\epsilon\right)^{2}}}-\frac{l}{\sqrt{l^{2}-L^{2}}}\right)
\end{aligned}
$$

where the constant is defined by $a \equiv \frac{3 \zeta(3)}{32 \pi^{3}}$. This result shows that the entropy behaves as $1 / \sqrt{\epsilon}$ at $\epsilon \rightarrow 0$ which corresponds to the ultraviolet divergence of the entropy. On the other hand, the distance of the brick wall from the horizon is related to the ultraviolet cutoff as

$$
\begin{aligned}
\tilde{\epsilon} & =\int_{r_{H}-\epsilon}^{r_{H}} \frac{d r}{\sqrt{g(r)}} \\
& =l\left(\frac{\pi}{2}-\sin ^{-1} \frac{l-\epsilon}{l}\right) .
\end{aligned}
$$


Then the entropy (15) is neatly represented in terms of the invariant cutoff (16) as follows,

$$
S=4 \pi a\left(\frac{1}{\sin \frac{\tilde{\epsilon}}{l}}-1\right)
$$

where we simply fix the infrared cutoff as $L=0$ without loss of generality since there does not exist any infrared divergence even though we consider massless scalar field. Note that the entropy is always positive in Eq. (17). If we choose the cutoff as

$$
\tilde{\epsilon}=l \sin ^{-1}\left(\frac{a}{a+l}\right)
$$

then the entropy is written by the perimeter law

$$
S=2 \cdot 2 \pi r_{+}
$$

Note that for $l \gg a$, the invariant cutoff is simply written as $\tilde{\epsilon} \approx a$ and it gives the entropy $S=4 \pi l$.

As a comment, at first sight the infrared regulator $L$ seems to be necessary in our massless field, however, it does not play an important role in DS space since our spacetime is bounded by the horizon and in some sense spacetime is surrounded by the black hole which is similar to the confined field in the cavity. Therefore, the finite volume of DS spacetime evaluated as $V(r)=2 \pi l^{2}\left[1-\sqrt{1-r^{2} / l^{2}}\right]$ removes infrared divergence in contrast to the asymptotically flat spacetime whose volume is infinite.

\section{ACKNOWLEDGMENTS}

This work was supported by the Korea Research Foundation (1997). 


\section{REFERENCES}

[1] J. D. Bekenstein, Lett. Nuovo Cimento 4, 737 (1972); Phys. Rev. D7, 2333 (1973); D9, 3292 (1974).

[2] S. W. Hawking, Commun. Math. Phys. 43, 199 (1975).

[3] G. 't Hooft, Nucl. Phys. B256, 727 (1985).

[4] M. Bañados, C. Teitelboim, and J. Zanelli, Phys. Rev. Lett. 69, 1849 (1992).

[5] S. Carlip and C. Teitelboim, Phys. Rev. D51, 622 (1995).

[6] S. Carlip, Phys. Rev. D51, 632 (1995).

[7] J. Maldacena and A. Strominger, gr-qc/9801096. 